\title{
Inhibition of Aurora Kinase A by Alisertib Reduces Cell Proliferation and Induces Apoptosis and Autophagy in HuH-6 Human Hepatoblastoma Cells
}

This article was published in the following Dove Press journal: OncoTargets and Therapy

\author{
Jingyi $\operatorname{Tan}^{1, *}$ \\ Wenfeng $\mathrm{Xu}\left(\mathbb{D}^{1, *}\right.$ \\ Lei Lei ${ }^{1}$ \\ Hui Liu' \\ Hong Wang ${ }^{2}$ \\ Xian Cao' \\ Man Xu' \\ 'Department of Pathology, Molecular \\ Medicine and Cancer Research Center, \\ Chongqing Medical University, Chongqing \\ 4000 16, People's Republic of China; \\ ${ }^{2}$ Institute of Life Science, Chongqing \\ Medical University, Chongqing 400016, \\ People's Republic of China \\ *These authors contributed equally to \\ this work
}

Purpose: Aurora kinase A (AURKA), which belongs to the serine/threonine protein kinase family, has been identified as a key driver of the genesis and progression of diverse tumors. The aim of this study was to determine the clinical significance of AURKA in patients with hepatoblastoma (HB) and the effect of inhibiting AURKA in the HB cell line HuH-6.

Methods: The expression of AURKA in HB tissue and adjacent normal liver tissue was detected by immunohistochemistry. Then, statistical analysis was performed to evaluate the association between AURKA expression and the clinicopathological characteristics of HB. The effect of AURKA knockdown on cell viability was assessed by CCK-8 assay. EdU and CCK-8 assays, Western blotting, flow cytometry, and transmission electron microscopy (TEM) were used to examine the effect of alisertib (ALS), a selective AURKA smallmolecule inhibitor, on the cell cycle, proliferation, apoptosis, and autophagy in HuH-6 human hepatoblastoma cells.

Results: The expression of AURKA was significantly higher in HB tissue than in adjacent normal tissue. Furthermore, high AURKA expression was associated with advanced Children's Oncology Group (COG) stage and tumor metastasis of HB. In vitro, AURKA knockdown significantly reduced the viability of HuH-6 cells, while ALS treatment significantly suppressed HuH-6 cell proliferation and induced G1-phase cell cycle arrest by reducing cyclin-D1 expression. Moreover, ALS promoted apoptosis and autophagy by decreasing the activity of p38 MAPK in HuH-6 cells.

Conclusion: High expression of AURKA is a potential predictor of poor prognosis in $\mathrm{HB}$ patients. AURKA knockdown reduced the viability of HuH-6 cells, and ALS treatment inhibited cell proliferation and induced apoptosis and autophagy via the p38 MAPK signaling pathway. Our results suggest that AURKA may be a novel therapeutic target and ALS a potential therapeutic drug for the treatment of $\mathrm{HB}$.

Keywords: prognosis, alisertib, proliferation, autophagy, apoptosis

\section{Introduction}

Although pediatric liver tumors are rare, hepatoblastoma (HB) is the fastest rising tumor and the most common hepatic malignant tumor globally in children under the age of 5. ${ }^{1,2}$ The incidence of HB in males is higher than in females. ${ }^{3} \mathrm{HB}$ is an embryonic tumor that arises from primary hepatoblasts and less differentiated cells, but its cause remains unclear. Currently, surgery resection and chemotherapy are the standard treatments for HB. Because of advances in the development of chemotherapeutic drugs, patients with low-risk HB now have an elevated event-free 5-year
Correspondence: Man Xu Department of Pathology, Molecular Medicine and Cancer Research Center, Chongqing Medical University, Chongqing 400016 , People's Republic of China Tel +86 I3I I0II 8908

Email2402316007@qq.com 
survival rate; however, patients with high-risk $\mathrm{HB}$ have a poor prognosis, with a 5 -year survival of between $21 \%$ and $28 \%{ }^{4,5}$ Therefore, it is of great importance that novel therapeutic targets are identified for the treatment of HB.

As conserved serine/threonine kinases, aurora kinases (A, B, and C) are critical for various mitotic events and maintenance of genomic integrity. ${ }^{6}$ Aurora kinase A (AURKA), the best-characterized member of the aurora kinase family, has important biological functions, including centrosome maturation and microfilament polymerization. AURKA has been shown to be highly expressed in a variety of malignant tumors, including bladder cancer, ${ }^{7}$ melanoma, ${ }^{8}$ and $\mathrm{HB}^{9}{ }^{9}$ Nonetheless, the clinical significance of AURKA expression remains unclear.

Alisertib (ALS), a novel AURKA-specific smallmolecule inhibitor, is currently undergoing clinical trials for different types of malignant tumors. However, the effect and underlying mechanism of ALS in HB is unknown.

In this study, we found that the expression levels of AURKA were higher in HB tissue than in adjacent nontumor tissue; moreover, the higher AURKA expression was correlated with Children's Oncology Group (COG) stage and metastasis in $\mathrm{HB}$ patients. We also found AURKA knockdown significantly suppressed the viability of HuH-6 cells, while ALS treatment affected the p38 MAPK pathway and induced G1-phase cell cycle arrest, apoptosis, and autophagy in HuH-6 cells. The results of our study suggested that AURKA may be a novel therapeutic target and ALS a potential therapeutic drug for the treatment of HB.

\section{Patients and Methods}

\section{Patients and Tissue Samples}

Thirty-three paraffin-embedded sections were obtained from the Children's Hospital of Chongqing Medical University. These sections contained 33 cases of HB tissues and 14 cases of adjacent liver tissues. The patients were between 1 and 144 months of age and had not received preoperative chemotherapy. Pathological diagnosis was confirmed by two pathologists. All procedures in this study were in accordance with the Declaration of Helsinki. Written informed consent was obtained from the parents or legal guardians of all the patients, and the study protocol was approved by the ethics committee of the Children's Hospital of Chongqing Medical University.

\section{Immunohistochemistry}

Paraffin-embedded tissue sections were dewaxed, rehydrated, and heat-treated with $0.01 \mathrm{M}$ citrate buffer $(\mathrm{pH}$ 6.0) for antigen retrieval. Immunohistochemical staining was then performed using Super Vision Kits (Boster, Wuhan, China). All procedures, including $\mathrm{H}_{2} \mathrm{O}_{2}$ blocking, BSA blocking, anti-AURKA antibody (1:300, Wanleibio Co., Ltd., Shenyang, China) staining at $4^{\circ} \mathrm{C}$ overnight and secondary antibody incubation were performed according to the manufacturer's protocol. Slides were stained with 3,3'-diaminobenzidine and counterstained with hematoxylin. Immunohistochemical evaluation was based on the intensity and extent of tumor cell staining. Staining intensity was graded as follows: 0 , negative; 1 , weakly positive; 2 , moderately positive; and 3 , strongly positive. Staining extent ( 0 to 4 ) was scored as follows: $0,<5 \% ; 1,6-25 \%$; $2,26-50 \% ; 3,51-75 \%$; and $4,>75 \%$ of positive cells. The final immunohistochemistry score (IS) was obtained by multiplying the intensity score by the staining extent score. An IS $<6$ was considered as low expression and an IS $\geq 6$ was considered as high expression. ${ }^{10}$

\section{Cell Line and Cell Culture}

The HuH-6 human hepatoblastoma cell line was purchased from Procell Life Science \&Technology Co., Ltd (Wuhan, China). The cells were cultured in DMEM (HyClone, Hudson, NH, USA) supplemented with $10 \%$ heatinactivated fetal bovine serum (FBS) and a $1 \%$ penicillin and streptomycin solution. The cells were cultured in an incubator with humidified air and $5 \% \mathrm{CO}_{2}$ at $37^{\circ} \mathrm{C}$. ALS powder was dissolved in dimethyl sulfoxide (DMSO) at a concentration of $100 \mathrm{mM}$ and stored at $-80^{\circ} \mathrm{C}$.

\section{Western Blot Analysis}

Total protein was extracted from HuH-6 cells using RIPA lysis buffer (Beyotime, Shanghai, China) containing phosphatase inhibitors (DingGuo, Beijing, China), and the protein was quantified by BCA Protein Quantification Kit (DingGuo, Beijing, China). Equal amounts of protein were separated by SDS-PAGE and transferred to formaldehyde-activated PVDF membranes. Subsequently, the membranes were blocked with 5\% skimmed milk at room temperature for 2 $\mathrm{h}$, and then incubated with primary antibodies against AURKA (1:1000), p-AURKA (1:1000), p38 MAPK (1:1000), p-p38 MAPK (1:1000), Bcl-2 (1:1000), Bcl-xL (1:1000), cleaved caspase-3 (1:500), cyclin-D1 (1:1000), LC3B (1:1000), and beta-actin (1:5000) at $4^{\circ} \mathrm{C}$ overnight. 
The bands were then incubated with horseradish peroxidaseconjugated secondary antibody (1:4000, Guangdong Saiguo Biotech Co., Ltd, China) at room temperature for $2 \mathrm{~h}$. Finally, enhanced chemiluminescence reagent (Beyotime, Shanghai, China) was used to visualize the protein bands. The data were analyzed using Image Lab software.

\section{siRNA Transfection}

siRNA targeting AURKA (AURKA-siRNA) and negative control siRNA (NC-siRNA) were purchased from RioboBio Co. Ltd (Guangzhou, China). The target sequence was TCTGGCTCTTAAAGTGTTA. HuH-6 cells were seeded in a 6 -well plate $\left(2 \times 10^{4}\right.$ cells/well) for $24 \mathrm{~h}$ and cell culture medium was substituted with DMEM without serum and penicillin and streptomycin. Subsequently, AURKA-siRNA and NC-siRNA were transfected into HuH-6 cells using Lipo Transfection Agent (C0526, Beyotime, Shanghai, China) according to the manufacturer's instructions. After $6 \mathrm{~h}$ of incubation, the medium was replaced with fresh complete medium.

\section{Cell Viability Assay}

The CCK-8 assay was used to detect cell viability. HuH-6 cells at the exponential phase were plated in 96-well plates $\left(5 \times 10^{3}\right.$ cells/well $)$ for $24 \mathrm{~h}$, and then treated with ALS and/ or siRNA. Cell viability was analyzed at 24,48 , and 72 h. Briefly, $10 \mu \mathrm{L}$ of CCK-8 solution (Apexbio, Houston, TX, USA) was added to each well. After incubation for $2 \mathrm{~h}$, the optical density (OD) at $450 \mathrm{~nm}$ was determined.

\section{Cell Proliferation Assay}

A BeyoClick EdU Cell Proliferation Assay Kit (Beyotime, Shanghai, China) was used to assess cell proliferation. $\mathrm{HuH}-$ 6 cells were seeded in 96-well plates at a density of $5 \times 10^{3}$ cells/well for $24 \mathrm{~h}$. After incubation with ALS for $48 \mathrm{~h}$, the cells were treated with $10 \mu \mathrm{M}$ EdU for $24 \mathrm{~h}$. Subsequently, the cells were fixed in $4 \%$ paraformaldehyde and permeabilized with $0.3 \%$ Triton X-100 for $10 \mathrm{~min}$. Nuclei were counterstained with Hoechst 33342. Finally, the specimens were visualized under a fluorescence microscope.

\section{Flow Cytometric Analysis of Apoptosis and the Cell Cycle}

HuH-6 cells were seeded in a 6-well plate $\left(2 \times 10^{4}\right.$ cells/ well) for $24 \mathrm{~h}$, and treated with ALS or 3-methyladenine (3-MA) for $48 \mathrm{~h}$. The cells were then harvested and cell cycle and apoptosis were evaluated by flow cytometry.

\section{Transmission Electron Microscopy (TEM)} Cells were fixed in $4 \%$ glutaraldehyde at $4{ }^{\circ} \mathrm{C}$ overnight, dehydrated before being post-fixed in $1 \% \mathrm{OsO}_{4}$ for $1.5 \mathrm{~h}$ at $4^{\circ} \mathrm{C}$, and then embedded in epoxy resin. The ultrathin sections were stained with uranyl acetate and lead citrate. Finally, the ultrastructure of HuH-6 cells was observed by TEM (JEM-2100Plus, Japan).

\section{Statistical Analysis}

SPSS 21.0 software was used for statistical analysis. The correlations between AURKA expression and HB clinicopathological features were analyzed using $\chi^{2}$ or Fisher's exact tests. The data for cellular experiments were presented as means \pm SD of at least three independent experiments and comparison between groups was determined by one-way ANOVA or the Student's $t$-test. $p<0.05$ was considered significant.

\section{Results}

\section{AURKA Was Highly Expressed in Hepatoblastoma Tissue}

AURKA protein expression in hepatoblastoma $(\mathrm{n}=33)$ and matched adjacent normal liver tissue $(n=14)$ was examined by immunohistochemistry. As shown in Figure 1, AURKA expression was mainly located in the cytoplasm. AURKA was highly expressed in 21 (63.64\%) of the $33 \mathrm{HB}$ cases, and AURKA expression was significantly higher in tumors than in adjacent tissues $(p<0.001)$.

\section{AURKA Expression Was Positively Correlated with HB Clinical \\ Aggressiveness}

The relationship between AURKA expression and HB clinicopathological parameters was analyzed. The results showed that high AURKA expression was significantly correlated with tumor metastasis $(p=0.0327)$ and COG stage ( $p=0.0163)$ but not sex, age, AFP level, or histological type (Table 1).

\section{AURKA Knockdown Inhibited the Viability of HuH-6 Cells}

To investigate the effect of AURKA on HuH-6 cells, we knocked down AURKA in HuH-6 cells using AURKAsiRNA. Western blot was performed to evaluate the efficiency of AURKA knockdown. As shown in Figure 2A and B, AURKA was effectively knocked down in HuH-6 cells by 

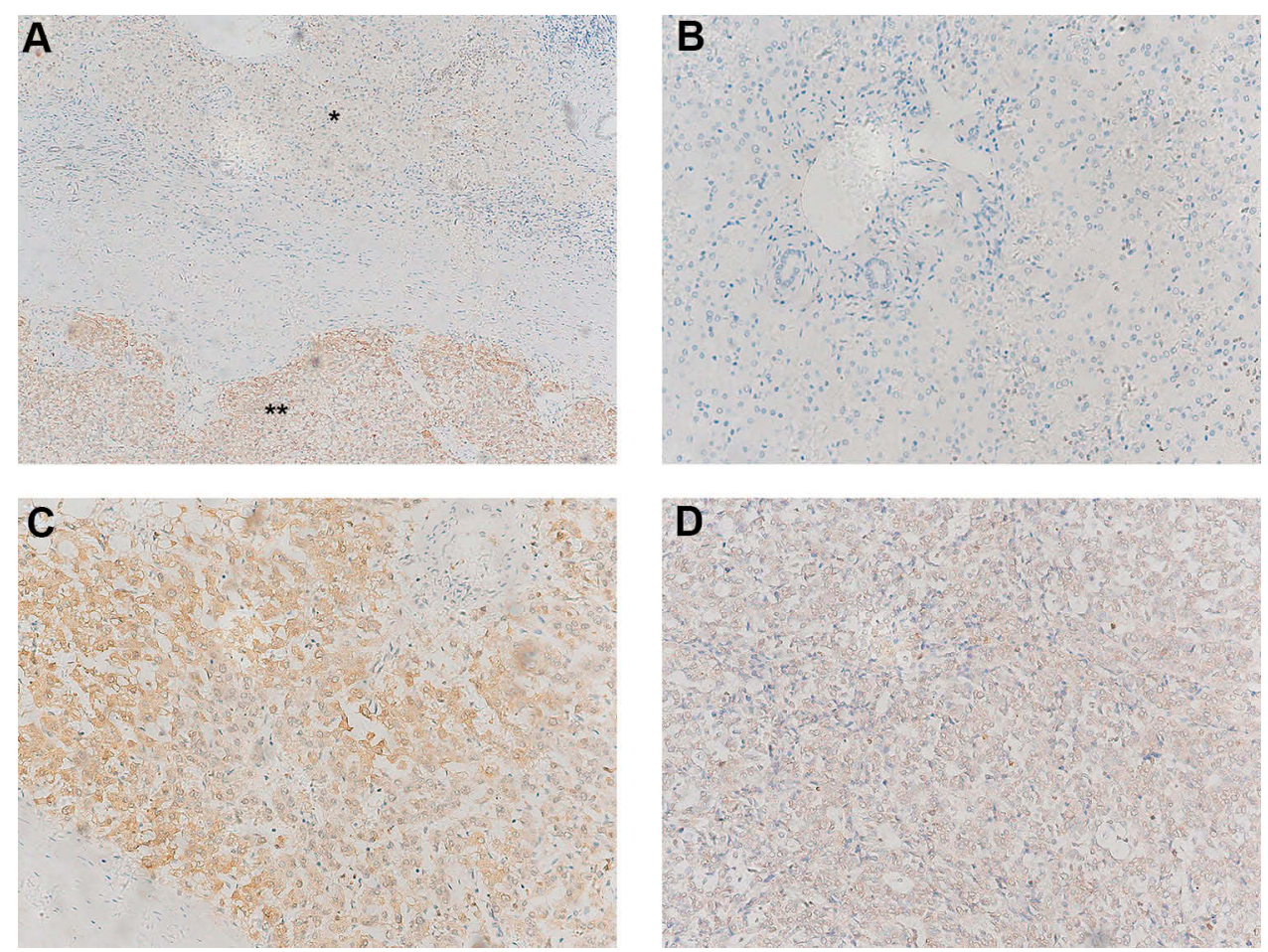

Figure I Expression levels of AURKA in hepatoblastoma (HB) tissue and adjacent normal tissue. (A) AURKA expression levels in HB tissue $(* *$, IS $=9)$ and adjacent normal live tissue $(*$, IS = I). (B) Negative AURKA staining in adjacent liver tissue (IS =0). (C) High AURKA expression in HB tissue (IS = 9). (D) Low expression of AURKA in HB tissue $(I S=2)$. (A) Original $\times 100$ magnification; (B-D) $\times 200$ magnification. IS, immunohistochemistry score.

AURKA-siRNA. We subsequently tested the effect of silencing AURKA on cell viability in HuH- 6 cells by CCK-8 assay. As shown in Figure 2C, cell viability was significantly suppressed in AURKA-siRNA-transfected cells compared with that in NC-siRNA cells. These results suggested that AURKA knockdown inhibits the viability of HuH-6 cells.

\section{ALS Treatment Reduced the Viability of HuH-6 Cells}

ALS has been identified as a novel AURKA-specific smallmolecule inhibitor. ${ }^{11}$ To test whether ALS affects HuH-6 cell viability, cells were treated with different concentrations $(1$, $5,12.5,25,50,75,100,150$, and $200 \mu \mathrm{M}$ ) of ALS for 48 h. The CCK-8 assay was then performed to evaluate cell viability. The results showed that ALS treatment inhibits the viability of HuH-6 cells in a concentration-dependent manner $\left[\mathrm{IC}_{50}=53.8 \mu \mathrm{M}\right.$, Figure $\left.3 \mathrm{~A}\right]$.

\section{AURKA Is a Specific Target of ALS in HuH-6 Cells}

To determine whether the ALS-induced effect on cell viability in HuH-6 cells was AURKA-specific, cells transfected or not with AURKA-siRNA were treated with $50 \mu \mathrm{M}$ ALS for
48 h. As shown in Figure 3B, ALS treatment significantly reduced the viability of NC-siRNA-transfected cells, but not that of AURKA-siRNA-transfected cells. These results suggested that ALS is specific for AURKA.

\section{ALS Inhibited the Proliferation of $\mathrm{HuH}-6$ Cells}

Since ALS could suppress HuH-6 cell viability, we further sought to determine whether ALS affects cell proliferation using the HuH-6 cell line. For this, we treated cells with 1, 5, or $50 \mu \mathrm{M}$ ALS for $48 \mathrm{~h}$. EdU assay results showed that the proliferation rate was significantly reduced after incubation with ALS at $1 \mu \mathrm{M}(p<0.001), 5 \mu \mathrm{M}(p<0.001)$, and $50 \mu \mathrm{M}$ $(p<0.001)$ for $48 \mathrm{~h}$ [Figure 3C and D], compared with the rate in the control group. These results suggested that ALS suppresses cell proliferation in HuH-6 cells.

\section{ALS Induced GI-Phase Arrest and Inhibited Cyclin-DI Expression in $\mathrm{HuH}-6$ Cells}

HuH-6 cells were treated with 1, 5, or $50 \mu \mathrm{M}$ ALS for $48 \mathrm{~h}$, and the effects on the cell cycle were evaluated by flow 
Table I Correlation Between the Expression of AURKA and the Clinicopathological Characteristics of 33 Hepatoblastoma Cases

\begin{tabular}{|c|c|c|c|}
\hline \multirow[t]{2}{*}{ Characteristics } & \multicolumn{2}{|c|}{ AURKA Expression } & \multirow[t]{2}{*}{$P$-value } \\
\hline & Low & High & \\
\hline Gender & & & 0.716 \\
\hline Male & 7 & 14 & \\
\hline Female & 5 & 7 & \\
\hline Age & & & 1.000 \\
\hline$\leq 8$ years & 10 & 18 & \\
\hline$>8$ years & 2 & 3 & \\
\hline $\operatorname{AFP}(\mathrm{ng} / \mathrm{mL})$ & & & 0.364 \\
\hline$<100$ & I & 0 & \\
\hline$\geq 100$ & 11 & 21 & \\
\hline Histology & & & 0.284 \\
\hline Epithelial & 12 & 18 & \\
\hline Mixed & 0 & 3 & \\
\hline COG stage & & & $0.016 *$ \\
\hline I-II & 7 & 3 & \\
\hline III-IV & 5 & 18 & \\
\hline Metastasis & & & $0.033^{*}$ \\
\hline Yes & 2 & 12 & \\
\hline No & 10 & 9 & \\
\hline
\end{tabular}

Note: ${ }^{*} p<0.05$.

Abbreviations: Mixed, mixed epithelial-mesenchymal; AFP, alpha-fetoprotein; COG, Children's Oncology Group.

cytometry and Western blotting. As shown in Figure 4A and $\mathrm{B}$, compared with the control group, ALS treatment increased the accumulation of HuH-6 cells in the G1 phase of the cell cycle from $33.05 \%$ to $43.50 \%$ and $45.3 \%$ at $5 \mu \mathrm{M}(p<0.05)$ and $50 \mu \mathrm{M}(p<0.001)$, respectively, and decreased the number of cells in the S phase from $58.96 \%$ to $50.56 \%, 43.99 \%$, and $26.45 \%$ at $1 \mu \mathrm{M}(p<0.01), 5 \mu \mathrm{M}(p<$ $0.001)$, and $50 \mu \mathrm{M}(p<0.001)$, respectively. The expression levels of cyclin-D1 were reduced in a concentrationdependent manner [Figure 4C and D]. These results showed that ALS likely inhibits the growth of HuH-6 cells via G1phase cell cycle arrest.

\section{ALS Promoted Apoptosis in HuH-6 Cells} HuH-6 cells were cultured with 1, 5, or $50 \mu \mathrm{M}$ ALS for $48 \mathrm{~h}$, and then the cell apoptosis rate was evaluated by flow cytometry. As shown in Figure 5A and B, compared with the control, ALS treatment markedly and dose-dependently increased the rate of apoptosis of HuH-6 cells from $23.57 \%$ to $32.99 \%, 38.56 \%$, and $42.67 \%$ at $1 \mu \mathrm{M}(p<0.05), 5 \mu \mathrm{M}$ $(p<0.01)$, and $50 \mu \mathrm{M}(p<0.001)$, respectively. Meanwhile, the expression of apoptosis-associated proteins was assessed by Western blotting. The expression of Bcl-2 and Bcl-xL was decreased in an ALS concentration-dependent manner, while that of cleaved caspase-3 was increased [Figure 5C and D]. Taken together, these results suggested that ALS treatment promotes apoptosis in HuH-6 cells.

\section{ALS Induced Autophagy in HuH-6 Cells}

Since ALS could promote apoptosis in HuH-6 cells, we further observed its effect on autophagy in HuH-6 cells by TEM and Western blotting. The expression level of LC3-II was greatly increased after treatment with ALS at 1,5 , or $50 \mu \mathrm{M}$ for

A

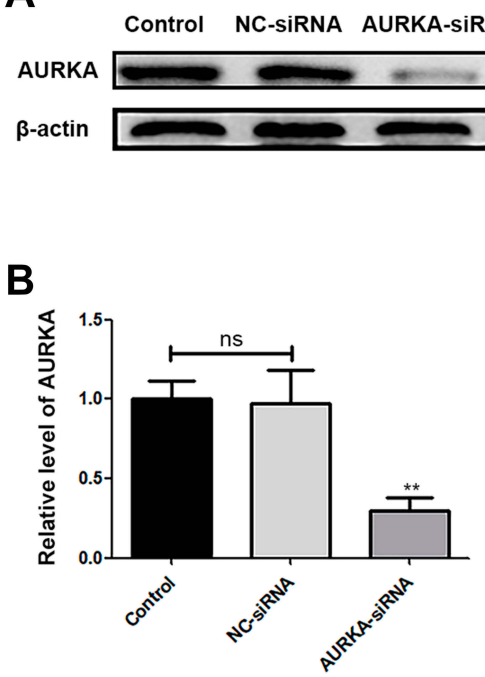

C

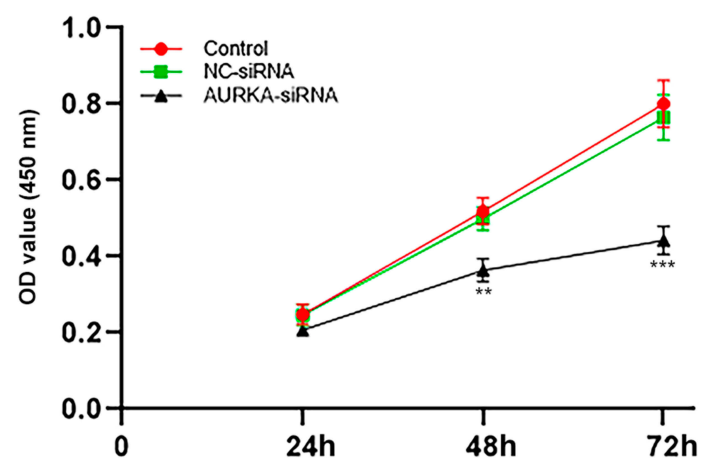

Figure 2 Effect of AURKA knockdown on the viability of HuH-6 cells. (A) Western blot assay of AURKA expression in HuH-6 cells at $72 \mathrm{~h}$ after transfection with AURKAsiRNA or negative control (NC)-siRNA ( $\mathrm{n}=3$ samples/group). (B) Quantification of (A). (C) CCK-8 assay for cell viability in response to AURKA knockdown in HuH-6 cells. Data are representative of three independent experiments. ${ }^{*} p<<0.01$, $*^{* *} p<0.00 \mathrm{I}$. ns, not significant. 

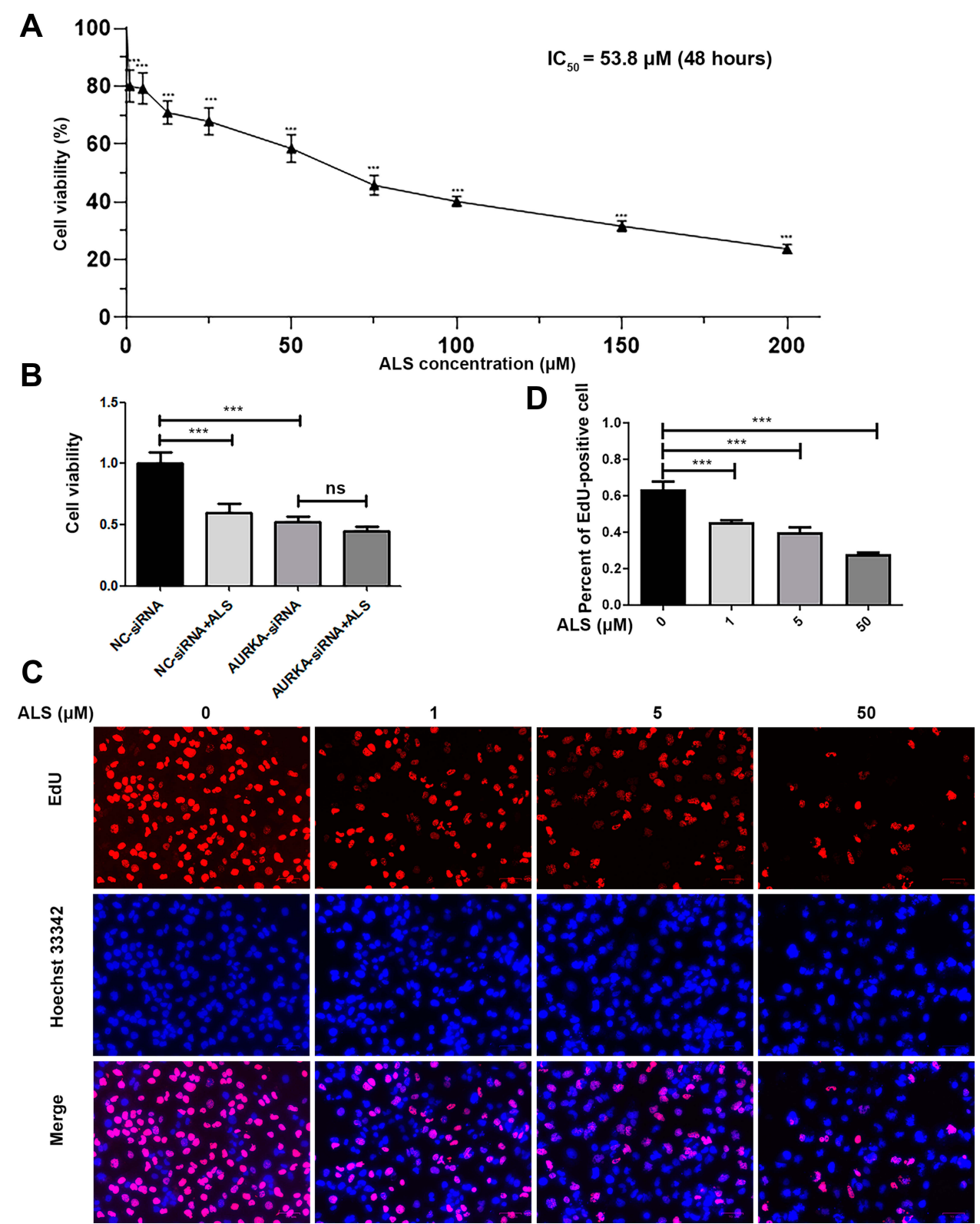

Figure 3 Effect of alisertib (ALS) on cell proliferation in HuH-6 cells. (A) CCK-8 assay for HuH-6 cell viability in response to incubation with ALS at I, 5, I2.5, 25, 50, 75, 100,150 , or $200 \mu \mathrm{M}$ for $48 \mathrm{~h}$. (B) CCK-8 assay for cell viability in siRNA-transfected HuH-6 cells treated or not with $50 \mu \mathrm{M}$ ALS for $48 \mathrm{~h}$. (C) EdU proliferation assay in cells treated with ALS at I, 5, or $50 \mu \mathrm{M}$ for $48 \mathrm{~h}$. (D) Quantification of EdU-positive HuH-6 cells after ALS treatment. Scale bars: $70 \mu \mathrm{m}$. Data are representative of three independent experiments. $*_{*} p<0.00 \mathrm{I}$. ns, not significant.

$48 \mathrm{~h}$ [Figure $5 \mathrm{C}$ and $\mathrm{D}]$. The results implied that ALS activates autophagy in HuH-6 cells. This effect was further verified by TEM. Treatment with ALS increased autophagosome accumulation in HuH-6 cells [Figure 5E]. To investigate the crosstalk between ALS-induced autophagy and apoptosis, HuH-6 cells were exposed to the autophagy inhibitor, 3-MA, and then assessed by Western blotting. Our data showed that the LC3-II protein level was decreased in cells cotreated with $50 \mu \mathrm{M}$ ALS and $5 \mathrm{mM} 3-\mathrm{MA}$ when compared with cells treated with $50 \mu \mathrm{M}$
ALS alone. However, 3-MA treatment did not affect LC3-II expression in untreated cells [Figure 6A and B]. We subsequently detected the effect of 3-MA on cell death. CCK-8 assay results showed that 3-MA further inhibited the viability of ALS-treated HuH-6 cells. However, 3-MA treatment had no effect on untreated control cells [Figure 6C]. Flow cytometric results suggested that the rate of apoptosis was significantly higher in cells cotreated with ALS and 3-MA than in cells treated with ALS alone $[p<0.05$; Figure $6 \mathrm{D}$ and E]. In 
A
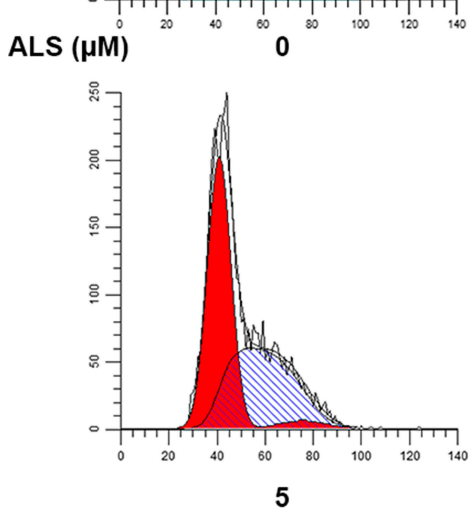

C

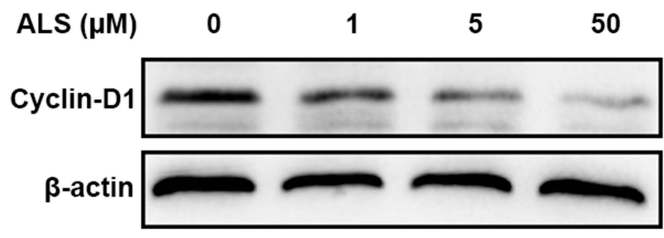

B
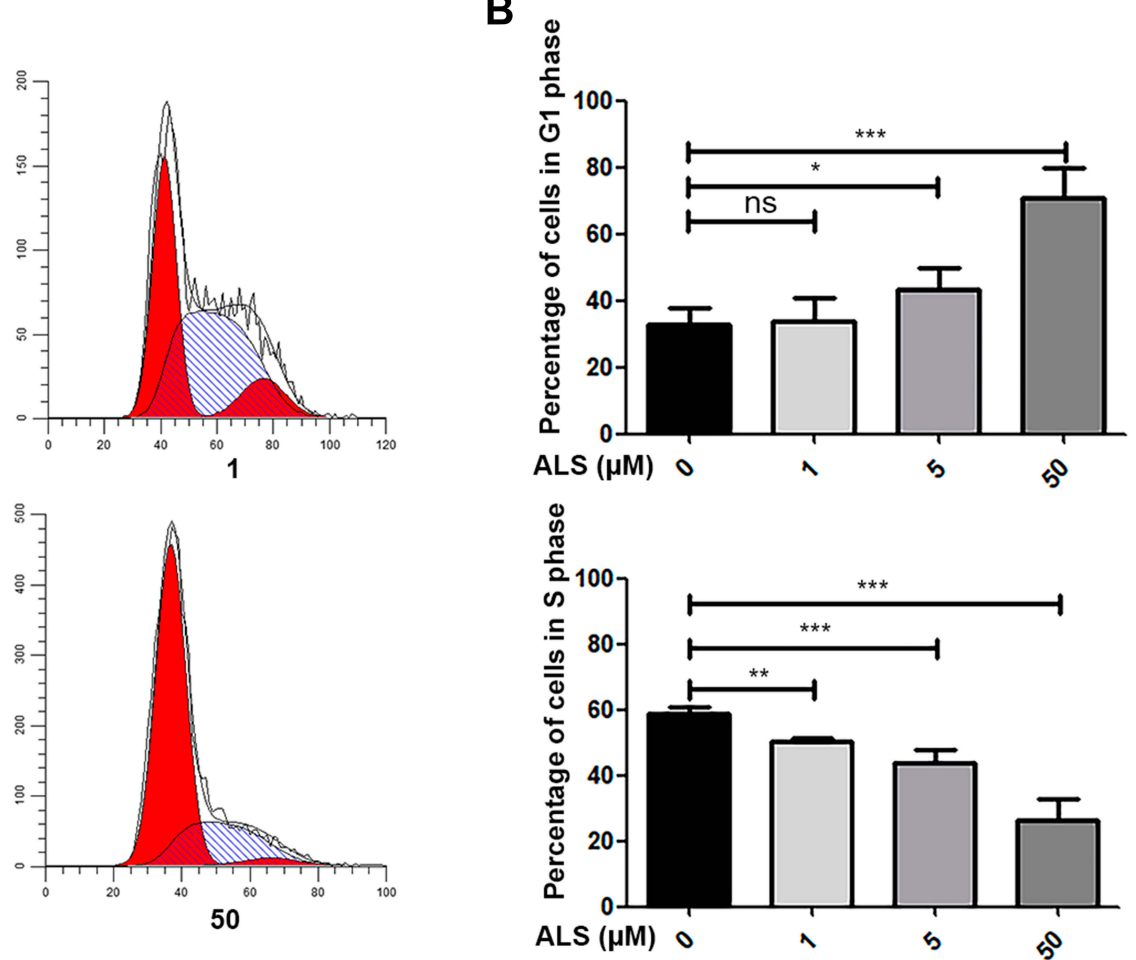

D

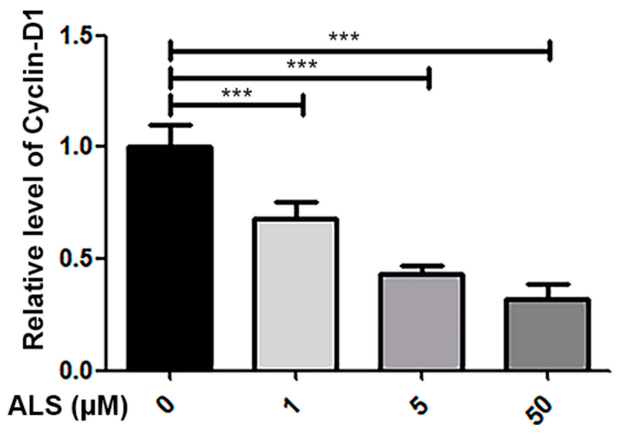

Figure 4 Effect of alisertib (ALS) on the cell cycle in HuH-6 cells. Cells were treated with ALS at I, 5, or $50 \mu M$ for $48 \mathrm{~h}$ and then subjected to flow cytometry and Western blotting. (A) Flow cytometry analysis of cell cycle stage distribution in $\mathrm{HuH}-6$ cells. (B) Quantification of the distribution of HuH-6 cells in the GI and $\mathrm{S}$ phases. (C) Western blot analysis of cyclin-DI levels in HuH-6 cells ( $n=3$ samples/group). (D) Quantification of (C). Data are representative of at least three independent experiments. ${ }^{*} p<0.05$, $*_{p}^{*} p<0.01$, ***p $<0.00 \mathrm{I}$. ns, not significant.

conclusion, inhibition of autophagy enhanced ALS-induced apoptosis in HuH-6 cells, indicating that autophagy may enhance cell survival.

\section{ALS Suppressed the Phosphorylation of p38 MAPK in HuH-6 Cells}

The p38 MAPK signaling pathway plays an important role in the growth and apoptosis of HB cells. We further evaluated the effect of ALS on p38 MAPK signaling. Western blot analysis showed that ALS treatment inhibited p-AURKA expression and reduced the levels of p38 MAPK phosphorylation [Figure $6 \mathrm{~F}$ and $\mathrm{G}$ ]. Combined, these data indicated the inhibitory effect of ALS on HuH-6 cells is likely exerted via regulation of p38 MAPK signaling.

\section{Discussion}

AURKA is shown to be highly expressed and to serve as a negative prognostic factor in multiple malignant tumors. $^{12-14}$ Zhang et $\mathrm{al}^{9}$ reported that aberrant expression of genes functioning upstream of AURKA leads to increased expression of AURKA and promotes tumor development in HB. In the present study, we found that AURKA expression is higher in HB tissue than in adjacent normal tissue. We further found that abnormally elevated 
A

ALS $(\mu \mathrm{M})$
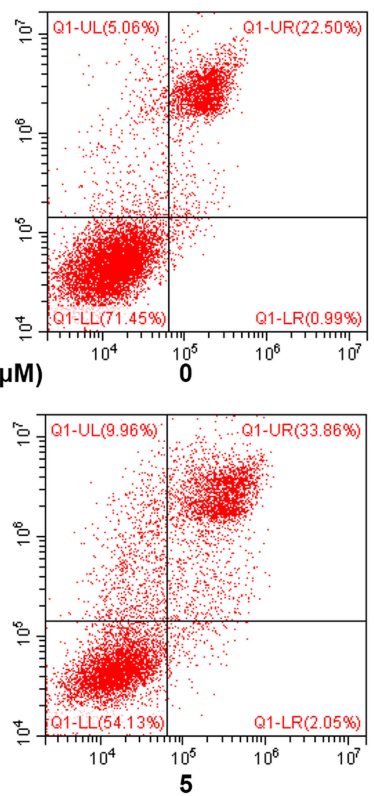

D
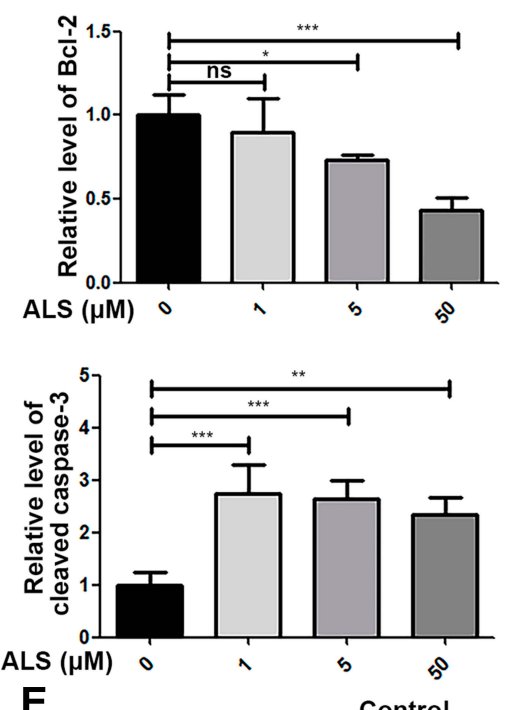

E

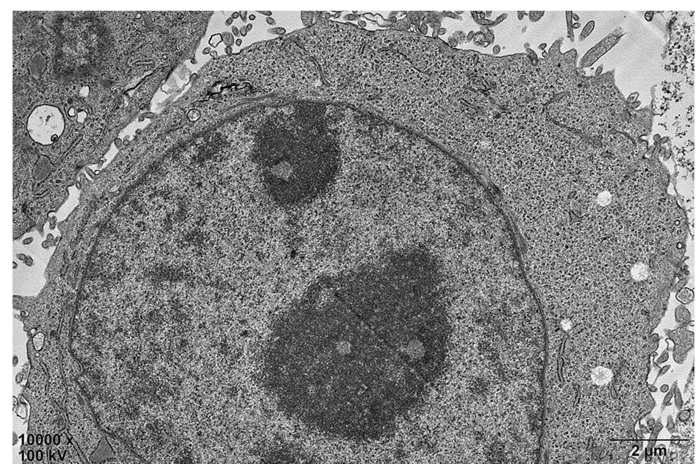

B
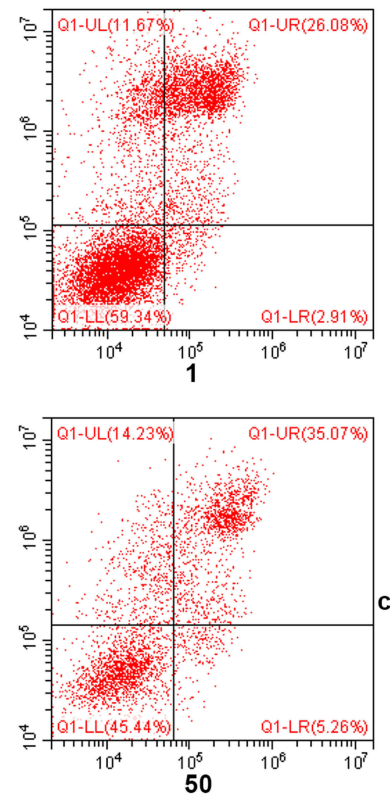

C
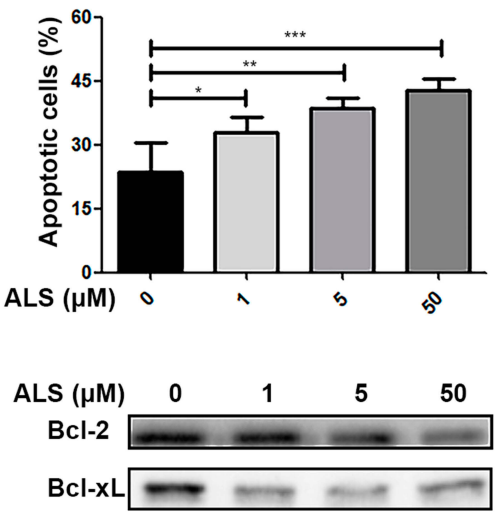
cleaved caspase-3
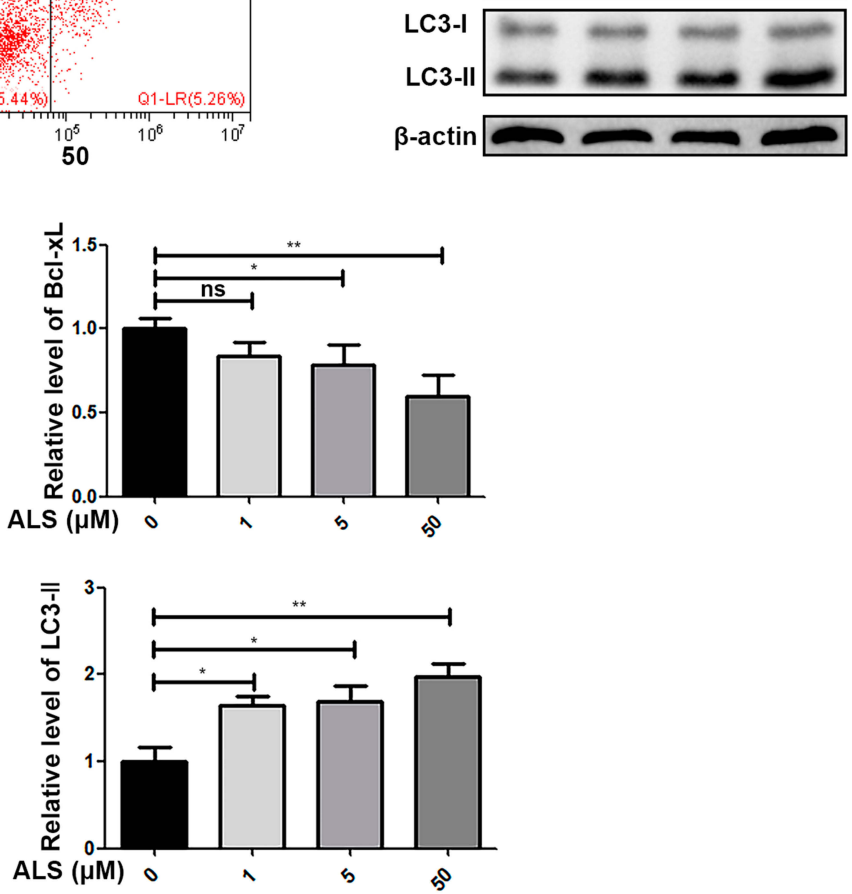

ALS

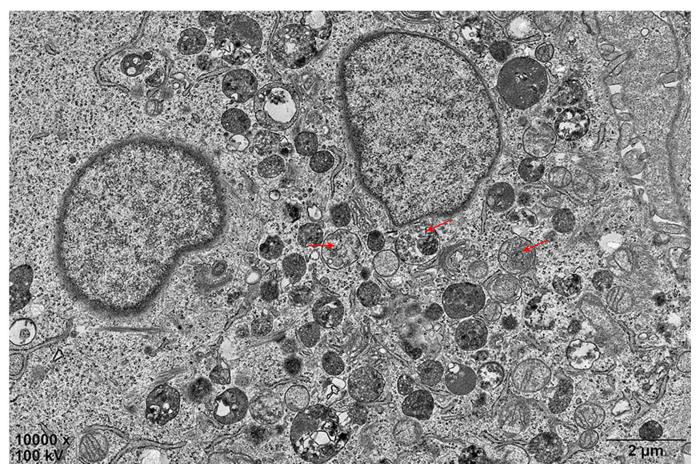

Figure 5 Effect of alisertib (ALS) on apoptosis and autophagy in HuH-6 cells. Cells were incubated with ALS at I, 5 , or $50 \mu M$ for 48 h and then subjected to flow cytometry and Western blot analysis. (A) Flow cytometric analysis of cell apoptosis. (B) Quantification of apoptosis in HuH-6 cells. (C) Western blot assay of the expression of Bcl-2, $\mathrm{Bcl}-\mathrm{xL}$, cleaved caspase-3, and LC3B ( $\mathrm{n}=3$ samples/group). (D) Quantification of (C). (E) Transmission electron micrographs of HuH-6 cells treated or not with $50 \mu M$ ALS for $48 \mathrm{~h}(\times 10,000)$. Red arrows indicate the autophagosome. Data are representative of three independent experiments. $*_{p}<0.05, * * p<0.0 \mathrm{I}, * * * p<0.00 \mathrm{I}$. ns, not significant. 
A

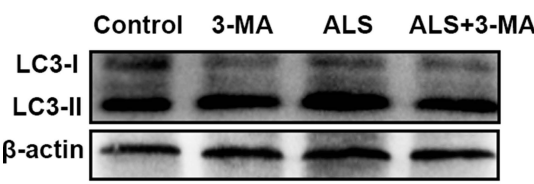

D
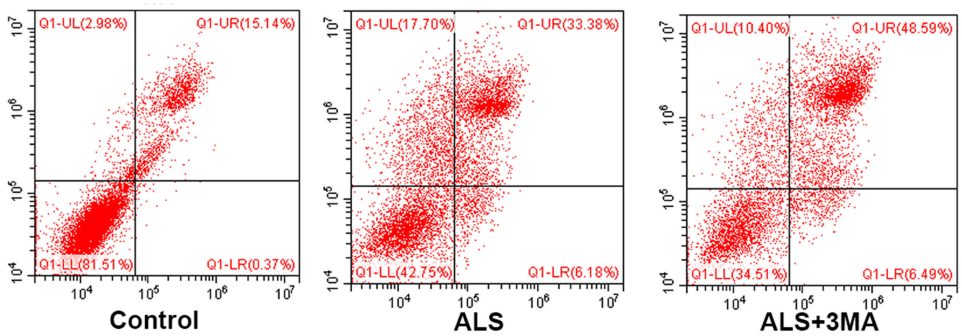

F

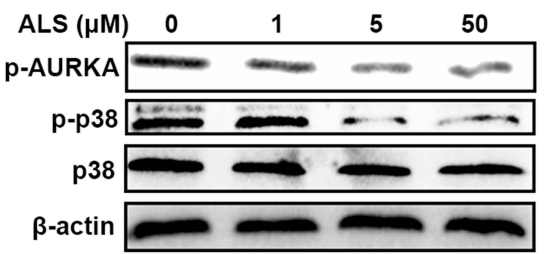

B

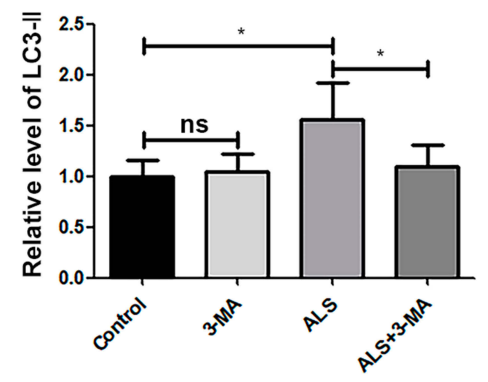

G

C
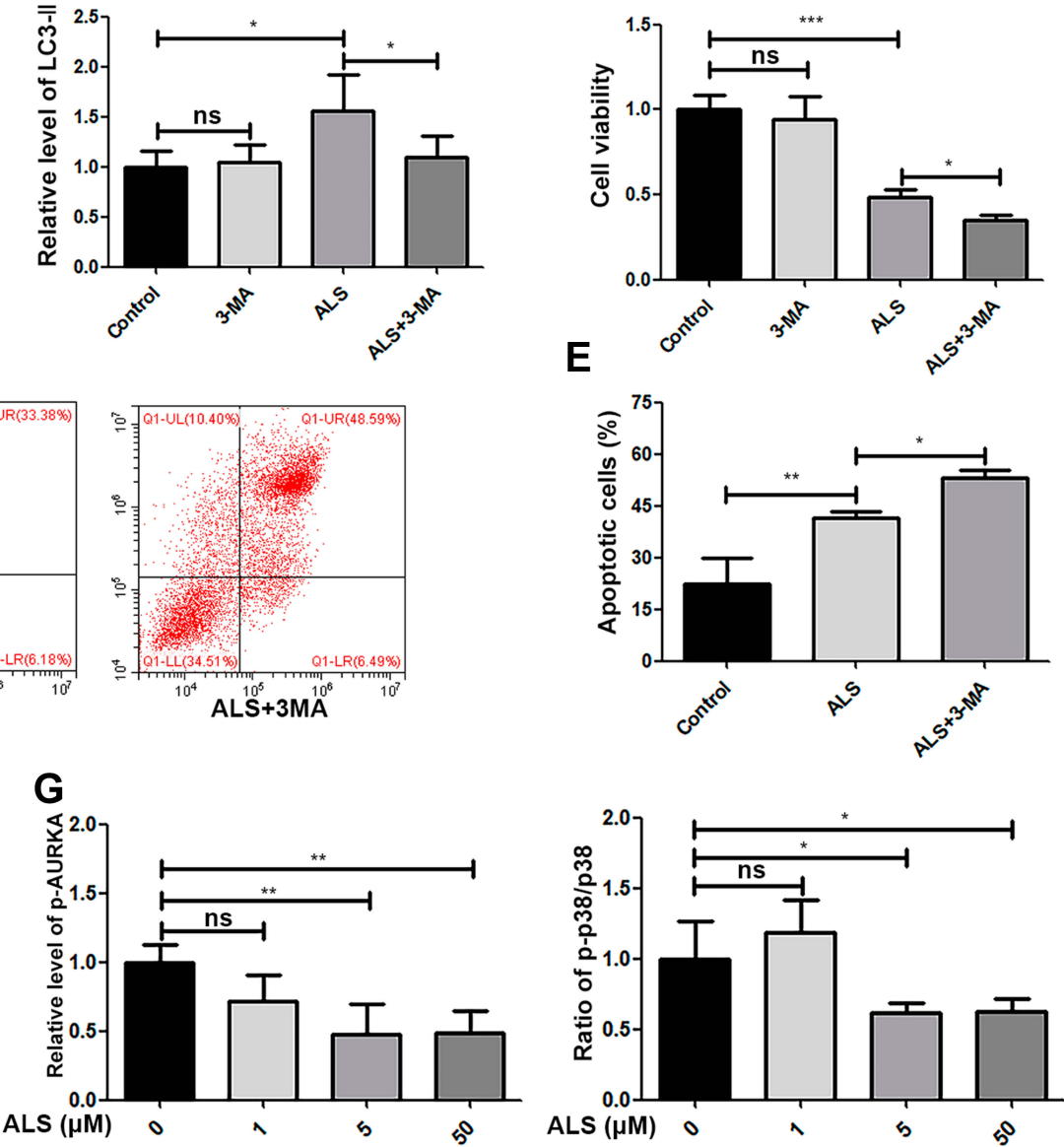

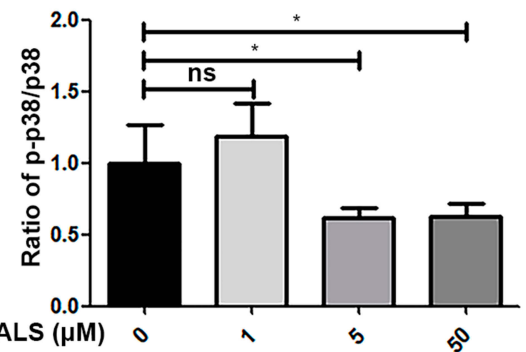

Figure 6 Effect of alisertib (ALS) on p38 signaling and crosstalk between ALS-induced autophagy and apoptosis in HuH-6 cells. Cells were treated with 5 mM 3-methyladenine (3-MA), $50 \mu \mathrm{M}$ ALS or $5 \mathrm{mM} \mathrm{3-MA}+50 \mu \mathrm{M}$ ALS for $48 \mathrm{~h}$. (A) Western blot assay of the level of LC3-\| in HuH-6 cells ( $\mathrm{n}=3 \mathrm{samples} / \mathrm{group})$. (B) Quantification of (A). (C) CCK-8 assay for HuH-6 cell viability. (D) Flow cytometric analysis of HuH-6 cell apoptosis. (E) Quantification of (D). (F) Western blot analysis of the levels of p-AURKA, p-p38 MAPK, and P38 MAPK in HuH-6 cells treated with ALS at I, 5, or $50 \mu M$ for $48 \mathrm{~h}$ ( $\mathrm{n}=3$ samples/group). (G) Quantification of the expression of $p$-AURKA and ratio of p-p38/p38 in HuH-6 cells incubated with I, 5, or $50 \mu \mathrm{M}$ ALS for $48 \mathrm{~h}$. Data are representative of at least three independent experiments. * $<0.05$, $* * p<0.01$, $* * * p<0.001$. ns, not significant.

AURKA expression is positively correlated with advanced COG stage and metastasis of $\mathrm{HB}$, and that AURKA knockdown suppresses HuH-6 cell viability. These results suggested that AURKA may be a potential therapeutic target and a predictor of prognosis in HB patients.

ALS is a selective small-molecule AURKA inhibitor that suppresses AURKA activation via its dephosphorylation at Thr288. ${ }^{11,15}$ Accumulating evidence has shown that ALS can suppress cell growth and promote autophagy and apoptosis in various tumor cell types. ${ }^{16-18}$ Consequently, we focused our experiments on determining the effect ALS on cell cycle progression and cell death in HuH-6 cells.

In this study, the CCK-8, EdU, and flow cytometry assay data indicated that ALS treatment markedly inhibits cell growth in a dose-dependent manner and induces G1- phase cell cycle arrest in HuH-6 cells. These results agree with those reported by Sun et $\mathrm{al}^{19}$ who demonstrated that knockdown of AURKA leads to cell cycle arrest at the G1-S transition and inhibition of cell proliferation in cervical cancer cells.

Autophagy is a key process in intracellular degradation and plays a major role in the maintenance of intracellular homeostasis. Although excessive autophagy can induce cell apoptosis, which makes targeting autophagy a promising direction for oncotherapy, the relationship between autophagy and apoptosis is complicated and controversial. Depending on circumstance and cell type, autophagy can promote either cell survival or programmed cell death. ${ }^{20}$ In our study, we found that ALS treatment induces autophagy and apoptosis in HuH-6 cells. To determine the crosstalk between ALS-induced autophagy and 
apoptosis, we suppressed autophagy through application of 3-MA. We observed a markedly higher rate of apoptosis when ALS-induced autophagy was inhibited by 3-MA compared with ALS treatment alone. This result indicated that autophagy protects cells from ALS-induced cytotoxicity. Similar findings have been documented for melanoma cells. $^{21}$

p38 MAPK signaling plays an essential role in the occurrence and development of tumors associated with cell growth and programmed cell death. ${ }^{22,23}$ Additionally, p38 MAPK signaling can inhibit autophagy in an mTORC1-independent manner, ${ }^{24}$ while the inhibitory effect of ALS in leukemic cells is shown to involve p38 MAPK signaling. ${ }^{25}$ To determine the mechanism underlying the ALS-induced inhibitory effect, we analyzed the activity of p38 MAPK signaling in HuH-6 cells. Our results showed that ALS treatment leads to the downregulation of $\mathrm{p}$-AURKA expression accompanied by inhibition of p38 MAPK phosphorylation. Several studies have shown that inactivation of p38 MAPK signaling leads to reduced expression of the anti-apoptosis proteins $\mathrm{Bcl}-2$ and Bcl-xL. ${ }^{26,27}$ Moreover, silencing p38 MAPK can inhibit the G1/S cell cycle transition in embryonic fibroblasts. $^{28}$ Shang et $\mathrm{al}^{21}$ demonstrated that ALS promotes apoptosis and autophagy in melanoma through deactivation of p38 MAPK. In this study, we found that ALS treatment exerts a similar effect on HB cells. These data indicated that the antitumor effect of ALS might be mediated via p38 MAPK signaling. However, further studies are needed to determine the interaction between AURKA and the p38 MAPK signaling pathway.

\section{Conclusion}

We have shown for the first time that high AURKA expression is associated with metastasis and advanced COG stage in patients with HB. We found that AURKA knockdown suppresses HuH-6 cell viability, and that ALS treatment inhibits proliferation and promotes apoptosis and autophagy in HuH-6 cells by regulating p38 MAPK signaling. This indicates that AURKA may be a prognostic biomarker and a potential therapeutic target and ALS is a potential therapeutic agent for the treatment of $\mathrm{HB}$.

\section{Acknowledgments}

This work was supported by the Department of Pathology, Chongqing Medical University.

\section{Disclosure}

The authors report no conflicts of interest in this work.

\section{References}

1. von Schweinitz D. Hepatoblastoma: recent developments in research and treatment. Semin Pediatr Surg. 2012;21(1):21-30. doi:10.1053/j. sempedsurg.2011.10.011

2. Hubbard AK, Spector LG, Fortuna G, Marcotte EL, Poynter JN. Trends in international incidence of pediatric cancers in children under 5 years of age: 1988-2012. JNCI Cancer Spectr. 2019;3(1): pkz007. doi:10.1093/jncics/pkz007

3. Herzog CE, Andrassy RJ, Eftekhari F. Childhood cancers: hepatoblastoma. Oncologist. 2000;5(6):445-453. doi:10.1634/ theoncologist.5-6-445

4. Meyers RL, Maibach R, Hiyama E, et al. Risk-stratified staging in paediatric hepatoblastoma: a unified analysis from the Children's Hepatic tumors International Collaboration. Lancet Oncol. 2017;18 (1):122-131. doi:10.1016/S1470-2045(16)30598-8

5. Lim IIP, Bondoc AJ, Geller JI, Tiao GM. Hepatoblastoma-the evolution of biology, surgery, and transplantation. Children (Basel, Switzerland). 2018;6(1).

6. Marumoto T, Zhang D, Saya H. Aurora-A - a guardian of poles. Nat Rev Cancer. 2005;5(1):42-50. doi:10.1038/nrc1526

7. Guo M, Lu S, Huang $\mathrm{H}$, et al. Increased AURKA promotes cell proliferation and predicts poor prognosis in bladder cancer. $B M C$ Syst Biol. 2018;12(Suppl 7):118. doi:10.1186/s12918-018-0634-2

8. Puig-Butille JA, VinyALS A, Ferreres JR, et al. AURKA overexpression is driven by FOXM1 and MAPK/ERK activation in melanoma cells harboring BRAF or NRAS mutations: impact on melanoma prognosis and therapy. J Invest Dermatol. 2017;137(6):1297-1310. doi:10.1016/j.jid.2017.01.021

9. Zhang Y, Zhao Y, Wu J, Liangpunsakul S, Niu J, Wang L. MicroRNA-26-5p functions as a new inhibitor of hepatoblastoma by repressing lin-28 homolog $\mathrm{B}$ and aurora kinase a expression. Hepatol Commun. 2018;2(7):861-871. doi:10.1002/hep4.1185

10. Zhou S, Venkatramani R, Gomulia E, Shillingford N, Wang L. The diagnostic and prognostic value of SALL4 in hepatoblastoma. Histopathology. 2016;69(5):822-830. doi:10.1111/his.13005

11. Manfredi MG, Ecsedy JA, Chakravarty A, et al. Characterization of Alisertib (MLN8237), an investigational small-molecule inhibitor of aurora A kinase using novel in vivo pharmacodynamic assays. Clin Cancer Res. 2011;17(24):7614-7624. doi:10.1158/1078-0432.CCR-11-1536

12. Shang X, Burlingame SM, Okcu MF, et al. Aurora A is a negative prognostic factor and a new therapeutic target in human neuroblastoma. Mol Cancer Ther. 2009;8(8):2461-2469. doi:10.1158/1535-7163.MCT-08-0857

13. Landen CN Jr., Lin YG, Immaneni A, et al. Overexpression of the centrosomal protein Aurora-A kinase is associated with poor prognosis in epithelial ovarian cancer patients. Clin Cancer Res. 2007;13 (14):4098-4104. doi:10.1158/1078-0432.CCR-07-0431

14. Ma Y, Yang J, Wang R, et al. Aurora-A affects radiosensitivity in cervical squamous cell carcinoma and predicts poor prognosis. Oncotarget. 2017;8(19):31509-31520. doi:10.18632/oncotarget.15663

15. Gorgun G, Calabrese E, Hideshima T, et al. A novel Aurora-A kinase inhibitor MLN8237 induces cytotoxicity and cell-cycle arrest in multiple myeloma. Blood. 2010;115(25):5202-5213. doi:10.1182/ blood-2009-12-259523

16. Ren BJ, Zhou ZW, Zhu DJ, et al. Alisertib induces cell cycle arrest, apoptosis, autophagy and suppresses EMT in HT29 and Caco-2 cells. Int J Mol Sci. 2015;17:1. doi:10.3390/ijms17010041

17. Baldini E, Tuccilli C, Prinzi N, et al. Selective inhibitors of aurora kinases inhibit proliferation, reduce cell viability and impair cell cycle progression in papillary thyroid carcinoma cells. $J$ Biol Regul Homeost Agents. 2015;29(4):793-803. 
18. Wang F, Li H, Yan XG, et al. Alisertib induces cell cycle arrest and autophagy and suppresses epithelial-to-mesenchymal transition involving $\mathrm{PI} 3 \mathrm{~K} / \mathrm{Akt} / \mathrm{mTOR}$ and sirtuin 1-mediated signaling pathways in human pancreatic cancer cells. Drug Des Devel Ther. 2015;9:575-601. doi:10.2147/DDDT.S75221

19. Sun JM, Yang LN, Xu H, Chang B, Wang HY, Yang G. Inhibition of Aurora A promotes chemosensitivity via inducing cell cycle arrest and apoptosis in cervical cancer cells. Am J Cancer Res. 2015;5 (3):1133-1145.

20. Reyjal J, Cormier K, Turcotte S. Autophagy and cell death to target cancer cells: exploiting synthetic lethality as cancer therapies. $A d v$ Exp Med Biol. 2014;772:167-188.

21. Shang YY, Yao M, Zhou ZW, et al. Alisertib promotes apoptosis and autophagy in melanoma through p38 MAPK-mediated aurora a signaling. Oncotarget. 2017;8(63):107076-107088. doi:10.18632/ oncotarget. 22328

22. Huang RH, Quan YJ, Chen JH, et al. Osteopontin Promotes Cell Migration And Invasion, And Inhibits Apoptosis And Autophagy In Colorectal Cancer by activating the p38 MAPK signaling pathway. Cell Physio Biochem. 2017;41(5):1851-1864. doi:10.1159/00047 1933
23. Gkouveris I, Nikitakis N, Sklavounou A. p38 expression and modulation of STAT3 signaling in oral cancer. Pathol Oncol Res. 2018. doi:10.1007/s12253-018-0405-9

24. Henson SM, Lanna A, Riddell NE, et al. p38 signaling inhibits mTORC1-independent autophagy in senescent human CD8(+) T cells. J Clin Invest. 2014;124(9):4004-4016. doi:10.1172/JCI75051

25. Fu Y, Zhang Y, Gao M, Quan L, Gui R, Liu J. Alisertib induces apoptosis and autophagy through targeting the AKT/mTOR/AMPK/ p38 pathway in leukemic cells. Mol Med Rep. 2016;14(1):394-398. doi: $10.3892 / \mathrm{mmr} .2016 .5249$

26. Flacke JP, Kumar S, Kostin S, Reusch HP, Ladilov Y. Acidic preconditioning protects endothelial cells against apoptosis through $\mathrm{p} 38$ and Akt-dependent Bcl-xL overexpression. Apoptosis. 2009;14 (1):90-96. doi:10.1007/s10495-008-0287-5

27. Zhang CL, Song F, Zhang J, Song QH. Hypoxia-induced Bcl-2 expression in endothelial cells via p38 MAPK pathway. Biochem Biophys Res Commun. 2010;394(4):976-980. doi:10.1016/j.bbrc.2010.03.102

28. Gong XW, Wei J, Li YS, Cheng WW, Deng P, Jiang Y. Effect of p38 mitogen-activated protein kinase gene knockout on cell proliferation of embryonic fibroblasts in mice. Chin Crit Care Med. 2008;20 (9):527-529.

\section{Publish your work in this journal}

OncoTargets and Therapy is an international, peer-reviewed, open access journal focusing on the pathological basis of all cancers, potential targets for therapy and treatment protocols employed to improve the management of cancer patients. The journal also focuses on the impact of management programs and new therapeutic agents and protocols on patient perspectives such as quality of life, adherence and satisfaction. The manuscript management system is completely online and includes a very quick and fair peer-review system, which is all easy to use. Visit http://www.dovepress.com/ testimonials.php to read real quotes from published authors. 